\title{
Crenças para lidar com tarefas de carreira em estudantes do ensino médio
}

\author{
Mara de Souza Leal, Lucy Leal Melo-Silva ${ }^{1}$ \\ Universidade de São Paulo, Ribeirão Preto-SP, Brasil \\ Maria Odilia Teixeira \\ Universidade de Lisboa, Lisboa, Portugal
}

\section{RESUMO}

O presente artigo visa a analisar a confiança com que estudantes do ensino médio lidam com tarefas de desenvolvimento da carreira, considerando as variáveis sexo, tipo de escola e nível socioeconômico. Os participantes $(N=241)$ da pesquisa são pessoas de ambos os sexos, de 17,5 anos em média e pertencentes a diferentes níveis socioeconômicos. Utilizou-se a versão brasileira do Inventário de Autoeficácia em Desenvolvimento da Carreira. A medida apresentou índices de precisão satisfatórios, com amplitude entre 0,65 e 0,75 . O efeito das variáveis sexo e tipo de escola não é estatisticamente significativo. O nível socioeconômico familiar tem efeito significativo, mostrando que os alunos de famílias com nível econômico mais elevado expressam mais confiança nas suas competências para planejamento de carreira, preparação para a procura de emprego e procura efetiva de emprego. São discutidas as propriedades psicométricas do CD-SEI e a necessidade de as intervenções para fortalecer as crenças dos estudantes.

Palavras-chave: autoeficácia; aprendizagem; desenvolvimento da carreira; orientação profissional; orientação vocacional.

\section{ABSTRACT - Beliefs when coping with career development tasks with highschool students}

This study is based on the Social Cognitive Theory and analyses the confidence with which highschool students, from the regular and technical schools cope with career development tasks. The variables analysed were sex, school type and family social-economic level. The participants $(N=241)$ were students aged 17.5 years of age on average male and female, coming from different socialecomonic levels. They answered the Brazilian version of the Career Development Self-Efficacy Inventory (CD-SEI-Br). The results showed satisfactory precision indices, with amplitude varying between .65 and .75 . The effect of gender and type of school is not statistically significant. However, the family socioeconomic level had a significant affect in that students from higher levels express more confidence when planning their career, preparing to find a job and actual finding a job. The psychometric properties of the CD-SEI are discussed, and the need for interventions to be addressed to strengthen the beliefs of students.

Keywords: self-efficacy; learning; career development; vocational guidance; career guidance.

\section{RESUMEN - Creencias de estudiantes secundarios para lidiar con tareas de carrera}

Este estudio analiza con qué confianza los estudiantes secundarios, regular y técnico, lidian con tareas de desarrollo de la carrera considerando las variables sexo, tipo de escuela y nivel socioeconómico familiar. Participantes $(N=241)$ de los dos sexos, con un promedio de 17,5 años, pertenecientes a diferentes niveles socioeconómicos respondieron a la versión brasileña del Inventario de Autosuficiencia en Desarrollo de la Carrera (CD-SEI- Br). La medida presentó en los resultados índices de precisión satisfactorios, con amplitud entre .65 y.75. El efecto del género y tipo de escuela no es estadísticamente significativa. Sin embargo, el nivel socioeconómico familiar tiene efecto significativo mostrando que alumnos de nivel más elevado expresan más confianza en sus competencias de planeamiento de carrera, de preparación para la búsqueda de empleo y para la búsqueda efectiva de empleo. Se discuten las propiedades psicométricas de CD-SEI, y la necesidad de intervenciones que deben reforzar las creencias de los estudiantes.

Palabras clave: autosuficiencia; aprendizaje; desarrollo profesional; orientación prefesional; orientación vocacional.

Nas últimas décadas, o construto da autoeficácia tem sido objeto de análise teórica e prática, contando com um corpo de estudos empíricos muito significativo em vários contextos do funcionamento humano, como é o caso da educação e da carreira. A autoeficácia é considerada um dos principais atributos do agenciamento humano, sendo um elemento central da teoria social cognitiva de Bandura (1986). No âmbito da carreira, Lent, Brown, e Hackett (1994), com base na teoria de Bandura (1977), explicam a formação dos interesses, a escolha e o desempenho por processos de aprendizagem, sendo dado especial relevância às crenças de autoeficácia, que 
se enraízam nas experiências, na aprendizagem vicariante, na persuasão verbal e nos estados emocionais. Essas fontes de autoeficácia possuem papel fundamental no desenvolvimento das competências para a vida, nomeadamente para a construção da carreira.

As pesquisas nesse domínio têm sido focadas sobretudo na tomada de decisão (Betz \& Luzzo, 1996; Taylor \& Betz, 1983), nos interesses (Betz \& Hackett, 1983; Lenox \& Subich, 1994; Lent, Brown, \& Larkin, 1984) e, atualmente, nos modelos de satisfação e bem-estar na carreira (Lent \& Brown, 2008). A maior parte desses estudos avalia a autoeficácia em diferentes comportamentos de carreira em relação às variáveis pessoais (sexo, idade e raça-etnia), às variáveis situacionais moderadoras dos processos de escolha (oportunidades de estudos e condições socioeconômicas). Existem também alguns estudos que analisam a autoeficácia em relação ao desempenho acadêmico, às aspirações, aos interesses e às próprias escolhas (Aguilera, 2013; Teixeira, 2007, 2008). Essas pesquisas têm contribuído para a validade dos modelos sociocognitivos de carreira (Lent, Brown, \& Hackett, 1994), explicando os processos da formação dos interesses, das escolhas e dos desempenhos acadêmicos em diferentes contextos culturais. No contexto brasileiro, contam-se algumas pesquisas sobre modelos sociocognitivos de carreira nomeadamente nos trabalhos de Leal (2013), Aguillera (2013); Noronha \& Ambiel (2008), Nunes \& Noronha (2009, 2011); Guerreiro-Casanova \& Polydoro (2011).

Apesar das pesquisas mencionadas, existem poucas investigações que examinem a especificidade das crenças nos processos do desenvolvimento da carreira, nas tarefas de desenvolvimento vocacional (Yuen, Gysbers, Chan, et al., 2004). Igualmente, constata-se que existem poucas medidas que operacionalizem esses conceitos, havendo alguns estudos com o Career Development Self-Efficacy Inventory (CD-SEI), quer na versão original (Yuen, Gysbers, Chan, et al., 2004, Yuen, Gysbers, Hui, et al., 2005), quer nas versões portuguesa (Lopes, 2010; Lopes \& Teixeira, 2012) e brasileira (Aguillera, 2013; Leal, 2013).

As crenças de autoeficácia são definidas por Bandura (1997, p. 3) como “... crenças nas próprias capacidades para organizar e executar cursos de ação requeridos para produzir certas realizações". Segundo Bandura (1997), as pessoas procuram se envolver em atividades que se considerem capazes de desempenhar satisfatoriamente e das quais obterão consequências positivas. Por esse motivo, as crenças de autoeficácia têm sido consideradas boas preditoras de comportamento e importantes para aquisição inicial de conhecimentos e habilidades (Pajares \& Olaz, 2008).

Ainda de acordo com Bandura (2001), uma crença fraca de autoeficácia desincentiva o indivíduo na persecução de comportamentos e pode até levá-lo a evitar comportamentos que acredite não ser capaz de realizar. Por outro lado, uma autoeficácia forte pode incentivar o indivíduo a se envolver em atividades mais desafiantes, como também pode auxiliá-lo no desenvolvimento de competências, desde que possua conhecimentos, habilidades mínimas e expectativas de resultados positivas em relação à tarefa executada. É importante pontuar, que a percepção sobre as crenças de autoeficácia (forte ou fraca) prediz o esforço e a persistência face às dificuldades no desempenho das tarefas.

O construto de autoeficácia articula os diferentes domínios da vida acadêmica dos estudantes, nomeadamente a aprendizagem e os projetos de carreira. No que se refere ao papel das crenças de autoeficácia na aprendizagem acadêmica, mais precisamente, Zimmerman (2000) verifica, pela análise de duas décadas de pesquisa, que são claras as evidências empíricas da importância do papel das crenças nos processos de aprendizagem, especialmente nos que envolvem autorregulação, como objetivos estabelecidos, automonitoramento, autoavaliação e uso de estratégias. Nesse sentido, alunos que se julgam mais eficazes são mais bem sucedidos nas tarefas autorregulatórias anteriormente citadas, e, consequentemente, nos seus processos de aprendizagem. As evidências também sugerem uma relação entre a avaliação positiva do desempenho acadêmico e o grau de confiança para lidar com as tarefas de carreira, designadamente o planejamento e a preparação para a procura de trabalho (Aguilera, 2013; Lopes, 2010). A pesquisa de Aguilera (2013) mostra relação positiva entre o próprio rendimento escolar e as crenças de confiança para lidar com as tarefas da carreira.

Em Portugal, nos estudos de Lopes (2010) sobre a autoeficácia no desenvolvimento da carreira e projetos vocacionais de estudantes com insucesso e risco de abandono escolar, os dados também indicam a relação entre a confiança em planejar a carreira e escolher a formação e o desempenho escolar. Uma das conclusões desse estudo é que os alunos com história de insucesso de famílias de poucos recursos percepcionam o seu desempenho escolar como médio ou ruim, possuem projectos de natureza qualificante e expressam menos confiança nas tarefas de planeamento de carreira e da selecção de formação (Lopes \& Teixeira, 2012). Em estudantes do ensino superior (Teixeira, 2008) também foi verificada a associação entre resultados escolares e percepção de capacidade para o sucesso acadêmico.

No âmbito da carreira, segundo Bandura (1993), as crenças de autoeficácia sobre aprendizagem e tarefas acadêmicas determinam o nível de aspiração e realizações acadêmicas dos alunos. As relações entre a autoeficácia de carreira e o nível de aspirações foram observadas nas pesquisas de Aguilera (2013), Lopes (2010) e de Yuen, Gysbers, Chan, et al. (2005), e os dados mostram que os estudantes com aspirações de carreira mais elevadas tendem a revelar resultados superiores no planejamento da carreira. Na pesquisa de Aguilera (2011), os dados evidenciam também uma relação estreita entre a autonomia 
dos jovens para a escolha e o grau de confiança percepcionado para gerir a carreira. Os jovens que consideram fatores pessoais nas suas decisões tendem a ser mais confiantes nas tarefas de desenvolvimento e de transição.

De acordo com Lent (2005), a autoeficácia e as expectativas de resultados - crenças formadas sobre as consequências dos comportamentos - ajudam a moldar os interesses dos indivíduos. Estudos realizados com amostras portuguesas evidenciam que os estudantes mais confiantes acerca das capacidades pessoais para obter sucesso acadêmico possuem também níveis de aspiração mais elevados e tendem a expressar interesses científicos mais acentuados (Teixeira, 2007, 2009).

A ligação entre as crenças de autoeficácia e os processos de aprendizagem social tem sido demonstrada por pesquisas em diferentes contextos geográficos e políticos. No contexto chinês, Huang e Hsieh (2011) verificaram que o nível socioeconômico dos pais era preditivo das crenças de autoeficácia para as decisões de carreira de adolescentes universitários. Por outro lado, Metheny e McWhirter (2013), no contexto norte-americano, não encontraram resultados significativos entre a relação direta do nível socioeconômico e da autoeficácia para as decisões de carreira de estudantes universitários. No estudo de Patel, Salahuddine, e O'Brien (2008), o nível socioeconômico também não contribuiu para a predição das crenças de autoeficácia para as decisões de carreira de adolescentes vietnamitas residentes em Washington, Estados Unidos. Na investigação realizada com estudantes portugueses do ensino superior (Teixeira, 2008), os dados tendem a confirmar a importância do ambiente familiar na construção das crenças de eficácia pessoal, salientando-se que, quanto mais elevado o nível de escolaridade das mães, maior o grau de autoconfiança dos filhos quanto à capacidade pessoal para obter sucesso acadêmico, à assertividade e à obtenção de apoio parental e comunitário.

Esses dados elucidam o significado das crenças no desenvolvimento dos percursos e trajetórias de vida e também apontam a necessidade de mais estudos sobre a autoeficácia no domínio da carreira. Lent et al. (2002) pontuam que a formação dos interesses se constitui em um processo contínuo que, apesar de mais fluido até o final da adolescência e início da vida adulta, ocorre ao longo do ciclo da vida. Os interesses podem ser modificados devido às circunstâncias, como mudanças tecnológicas, perda de emprego, nascimentos dos filhos, acidentes, entre outras. Os autores consideram ainda que outras variáveis relativas à pessoa ou ao contexto influenciam a formação dos interesses e de outros resultados de carreira, como sexo, raça-etnia, estado de saúde, genética e condições econômicas. Quanto ao sexo, especificamente, os referidos autores salientam a influência de pais e professores no desenvolvimento dos interesses em função das expectativas de gênero adequadas a diferentes culturas. Também os dados das pesquisas realizadas com amostras de estudantes em Portugal têm demonstrado que as crenças de autoeficácia diferem em função do gênero e das decisões dos estudantes, especificamente das escolhas de cursos no ensino superior ou de cursos técnicos e profissionalizantes (Teixeira, 2007, 2009; Teixeira, Barreira, Faria, Baptista, \& Pimentel, 2011).

$\mathrm{Na}$ sequência das investigações realizadas em língua portuguesa, a atual pesquisa também dá continuidade ao processo de validação da versão brasileira do CD-SEI, iniciado por Aguilera (2013), para o contexto brasileiro. Neste estudo examinam-se as qualidades psicométricas da medida e analisam-se as crenças de competências de carreira em alunos do ensino médio.

\section{Método}

\section{Participantes}

Participaram deste estudo 241 alunos do terceiro ano do ensino médio público de duas escolas de uma cidade do interior do estado de São Paulo. Os alunos frequentavam o ensino médio regular $(60 \%)$ e o ensino técnico nas áreas de ciências exatas, ciências humanas e ciências biológicas (40\%). As idades dos participantes variavam de 16 a 20 anos $(M=17,5 ; D P=0,89)$, sendo $67 \%$ do sexo feminino e $33 \%$ do sexo masculino. $72 \%$ dos participantes classificam-se nos níveis A e B, e $28 \%$, nos níveis $\mathrm{C}$ e $\mathrm{D}$, segundo o Critério de Classificação Econômica Brasil (ABEP, 2012), instrumento que mensura o poder de compras de famílias em meio urbano e as alocam em oito classes decrescentes em função do poder aquisitivo.

\section{Instrumentos}

Foi utilizada a versão brasileira do Career Development Self-Efficacy Inventory (CD-SEI) (Aguillera, 2013), cuja versão original é chinesa, de Hong Kong (Yuen, Gysbers, Chan, et al., 2004, Yuen, Gysbers, Hui, et al., 2005). O CD-SEI avalia a confiança dos estudantes para lidarem com as tarefas de carreira, designadamente as que caracterizam a fase de exploração (Super, 1990). De acordo com os autores (Yuen, Gysbers, Chan, et al., 2004), as escalas do CD-SEI (Planejamento de Carreira, Questões de Gênero na Carreira, Informação para Escolha Profissional, Preparação para Busca de Emprego, Procura de Emprego e Definição de Objetivos) representam as competências necessárias para a transição da escola para o mundo do trabalho.

O instrumento contém 24 itens que formam as seis escalas. A escala de Planejamento de Carreira inclui conteúdos que incidem na congruência entre o autoconhecimento e as alternativas de carreira (por exemplo, equilibrar interesses e perspectivas futuras). A escala Questões de Gênero na Carreira foca conteúdos que relacionam a carreira com o gênero (por exemplo, lidar com obstáculos, críticas e pontos de vista opostos, escolher uma carreira desempenhada principalmente por pessoas do sexo 
oposto). A escala Informação para Escolha Profissional inclui conteúdos sobre a confiança com que os jovens usam os recursos exteriores (por exemplo, compreender um programa de formação profissional antes de se matricular). A escala Preparação para Busca de Emprego incide sobre o grau de confiança nas tarefas de inserção profissional, nomeadamente na procura de emprego (por exemplo, dominar técnicas gerais para participar de entrevista de emprego ou estágio, como aparência, modos de falar etc.). Os conteúdos da escala Procura de Emprego focalizam as competências na procura efetiva de emprego (por exemplo, procurar trabalho adequado de acordo com meus interesses e capacidades). A escala Definição de Objetivos de Carreira incide sobre a confiança para estabelecer objetivos e empreender estratégias para os atingir (por exemplo, melhorar constantemente estudos e plano de carreira para trabalhar em direção a meus objetivos profissionais e de carreira). O CD-SEI apresenta um escore total das seis escalas. O grau de confiança do jovem em suas competências é mensurado por respostas a questões do tipo "Estou confiante de que sou capaz de...", em uma escala de Likert de seis pontos de 1, "Nada confiante", a 6, "Muito confiante".

Nos estudos com a escala original, a análise da precisão da medida revela bons índices de consistência interna. $\mathrm{Na}$ totalidade da escala, o coeficiente alfa é de 0,95 , e nas subescalas, entre 0,77 e 0,82 (Yuen, Gysbers, Chan, et al., 2004,). Na versão estudada por Aguillera (2013), em amostras brasileiras, nas seis escalas, os coeficientes alfa são de 0,69 a 0,78 , e, na escala total, 0,94 . Nas amostras portuguesas, numa amostra do $7^{\circ}$ ano de escolaridade, os coeficientes alfas oscilam entre 0,70 e 0,79 (Lopes, 2010; Lopes \& Teixeira, 2012), enquanto em amostras do ensino secundário os alfas variam entre 0,48 e 0,72 (Teixeira et al., 2011), observando-se o coeficiente mais baixo $(0,48)$ na escala Questões de género na Carreira.

O Critério de Classificação Econômica Brasil é um instrumento elaborado pela Associação Brasileira de Empresas de Pesquisa (ABEP, 2012), que objetiva medir o poder de compras de famílias em meio urbano. A posse de bens, como determinados produtos eletrodomésticos, automóveis, quantidade de banheiros, e serviços, como empregada mensalista, além do grau de instrução educacional do chefe da família, alocam o indivíduo em oito classes decrescentes, em função do poder aquisitivo (A1, A2, B1, B2, C1, C2, D e E), sendo que cada classe apresenta um valor bruto estimado de renda média familiar em reais. Para fins de análise deste estudo, foram considerados de níveis socioeconômico familiar alto os participantes classificados das classes A e $\mathrm{B}$, e de nível socioeconômico familiar baixo, os das classes C e D.

\section{Procedimentos}

Após a autorização dos diretores das escolas e aprovação pelo Comitê de Ética em Pesquisa de uma Instituição de Ensino Superior pública, a aplicação dos instrumentos foi realizada em sala de aula e de forma coletiva. A aplicação do instrumento foi realizada por uma equipe treinada, formada por cinco psicólogas e uma estudante do último ano de Psicologia, e teve a duração média de 10 minutos. Consideraram-se os cuidados éticos necessários quanto ao esclarecimento dos objetivos da pesquisa, a natureza voluntária da tarefa, a confidencialidade dos dados e a possibilidade de desistência a qualquer momento. Ressalta-se que somente os estudantes que entregaram o Termo de Consentimento Livre e Esclarecido assinado participaram da pesquisa, sendo necessária a autorização dos pais no caso dos estudantes menores de 18 anos.

\section{Análise de Dados}

Os dados foram sistematizados no package estatístico SPSS, versão 15.0, e foram usados procedimentos de estatísticas descritivas e inferenciais. No propósito de verificar os índices psicométricos do instrumento, foram estimados índices da consistência interna e da estrutura interna da medida CD-SEI.

\section{Resultados}

A consistência interna da medida é estimada pelo coeficiente alfa de Cronbach. Os coeficientes variam de 0,65 (Questões de Gênero na Carreira) a 0,75 (Preparação para Busca de Emprego). Na escala total, o coeficiente situa-se em 0,92, e a mediana em 0,71 (Tabela 1).

Tabela 1

Coeficientes de Alfa de Cronbach para as Dimensões e Escore Total do CD-SEI-Br

\begin{tabular}{lc}
\hline \multicolumn{1}{c}{ Dimensões do CD-SEI-Br } & Alfa de Cronbach \\
\hline Planejamento de Carreira & 0,67 \\
Questões de Gênero na Carreira & 0,65 \\
Informação para Escolha Profissional & 0,69 \\
Preparação para Busca de Emprego & 0,75 \\
Procura de Emprego & 0,73 \\
Definição de Objetivos de Carreira & 0,74 \\
Escore total & 0,92 \\
\hline
\end{tabular}


A Tabela 2 apresenta a matriz de correlação de Pearson das escalas do CD-SEI-Br. A amplitude dos coeficientes varia entre 0,49 (Preparação para Busca de Emprego e Questões de Gênero na Carreira) e 0,73 (Procura de Emprego e Preparação para Busca de Emprego), situando-se a mediana dos coeficientes em 0,66.

Tabela 2

Matriz de Correlação (Pearson) das Dimensões do CD-SEI-Br

\begin{tabular}{|c|c|c|c|c|c|c|c|}
\hline & & & & S̃es do & & & \\
\hline & PC & QGC & IEP & $\mathrm{PBE}$ & $\mathrm{PE}$ & DOC & Escore \\
\hline PC & - & & & & & & \\
\hline QGC & $0,54^{* *}$ & - & & & & & \\
\hline IEP & $0,62^{* *}$ & $0,59^{* *}$ & - & & & & \\
\hline $\mathrm{PBE}$ & $0,57^{* *}$ & $0,49^{* *}$ & $0,66^{* *}$ & - & & & \\
\hline $\mathrm{PE}$ & $0,68^{* *}$ & $0,55^{* *}$ & $0,66^{* *}$ & $0,73^{* *}$ & - & & \\
\hline DOC & $0,70^{* *}$ & $0,61^{* *}$ & $0,70^{* *}$ & $0,60^{* *}$ & $0,68^{* *}$ & - & \\
\hline Escore Total & $0,82^{* *}$ & $0,75^{* *}$ & $0,85^{* *}$ & $0,83^{* *}$ & $0,87^{* *}$ & $0,86^{* *}$ & - \\
\hline
\end{tabular}

Nota. PC (Planejamento de Carreira); QGC (Questões de Gênero na Carreira); IEP (Informação para Escolha Profissional); PBE (Preparação para Busca de Emprego); PE (Procura de Emprego); DOC (Definição de Objetivos de Carreira)

${ }^{* *} p \leq 0,01$

A comparação das médias dos resultados do CDSEI em função do nível socioeconômico familiar ( $t$ de Student), conforme Tabela 3, mostra que os estudantes com famílias de nível socioeconômico mais elevado apresentam resultados médios significativamente superiores aos dos seus colegas nas escalas Planejamento de Carreira $(p<0,01)$, Preparação para Busca de Emprego $(p<0,05)$ e Procura de Emprego $(p<0,05)$ e ainda no escore total $(p<0,05)$.

$\mathrm{Na}$ análise da variabilidade dos resultados em função das variáveis sexo e tipo de escola (regular ou técnica) não há diferenças significativas entre os resultados médios dos grupos.

Tabela 3

Resultados da Autoeficácia em Desenvolvimento de Carreira (CD-SEI-Br) em Função do Nível Socioeconômico Familiar

\begin{tabular}{|c|c|c|c|c|c|c|c|c|c|c|}
\hline \multirow[t]{2}{*}{ Dimensões } & \multicolumn{4}{|c|}{$\begin{array}{c}\text { Nível Socioeconômico } \\
\text { Familiar AB }(\mathrm{N}=174)\end{array}$} & \multicolumn{4}{|c|}{$\begin{array}{c}\text { Nível Socioeconômico } \\
\text { Familiar CD }(\mathrm{N}=67)\end{array}$} & \multirow[t]{2}{*}{$t$} & \multirow[t]{2}{*}{$p$} \\
\hline & Mín. & Máx. & M & DP & Mín. & Máx. & M & $D P$ & & \\
\hline Planejamento de Carreira & 2 & 6 & 4,94 & 0,61 & 3 & 6 & 4,70 & 0,61 & 2,67 & 0,01 \\
\hline Questões de Gênero na Carreira & 4 & 6 & 5,05 & 0,58 & 3 & 6 & 4,86 & 0,75 & 1,88 & 0,06 \\
\hline Informação para Escolha Profissional & 3 & 6 & 4,57 & 0,62 & 2 & 6 & 4,40 & 0,76 & 1,73 & 0,08 \\
\hline Preparação para Busca de Emprego & 2 & 6 & 4,60 & 0,76 & 2 & 6 & 4,37 & 0,78 & 2,08 & 0,04 \\
\hline Procura de Emprego & 3 & 6 & 4,80 & 0,64 & 3 & 6 & 4,60 & 0,72 & 2,08 & 0,04 \\
\hline Definição de Objetivos de Carreira & 3 & 6 & 4,81 & 0,66 & 2 & 6 & 4,68 & 0,70 & 1,35 & 0,18 \\
\hline CD-SEI total & 3 & 6 & 4,80 & 0,53 & 3 & 6 & 4,60 & 0,61 & 2,41 & 0,02 \\
\hline
\end{tabular}

Nota. $p<0,05$

\section{Discussão}

Os resultados mostram coeficientes considerados moderados, mas favoráveis, de consistência interna da medida, variando de 0,65 a 0,75 nas seis escalas, e de 0,92 no escore total. Esses resultados são relativamente próximos aos observados em amostras brasileiras (Aguillera, 2013) e portuguesas (Teixeira et al., 2011). Salienta-se que, na versão original, os coeficientes são mais elevados. No seu conjunto, os coeficientes encontrados nos estudos brasileiros, portugueses e chinês são favoráveis à homogeneidade da medida.

A matriz de correlações apresenta características de multicolinearidade, evidenciando uma associação elevada entre os resultados das seis escalas. Esses dados assemelham-se aos encontrados em outros estudos na versão em língua portuguesa do Brasil (Aguillera, 2013) e de Portugal (Lopes, 2010; Teixeira et al., 2011) e na versão original do inventário (Yuen, Gysbers, Chan, et al., 2005), sendo sugestivos de uma dimensão geral subjacente à autoeficácia 
para as tarefas da carreira na fase de exploração. Apesar da construção do CD-SEI partir do pressuposto de que existem seis dimensões representativas das competências para lidar com as tarefas de desenvolvimento durante a adolescência, a análise fatorial confirmatória dos resultados dos itens da versão original do instrumento indica seis fatores primários e um fator geral. Contudo, nos resultados das escalas, as correlações são próximas às obtidas neste estudo (Yuen, Gysbers, Chan, et al., 2004). Na amostra brasileira (Aguilera, 2013), a análise fatorial exploratória dos resultados dos itens tende a proporcionar uma solução de 3 fatores, em que um reúne os aspectos de planejamento de carreira e da definição de objetivos; outro, as questões de género e a informação; e um terceiro, as duas escalas relacionadas à procura e à entrada no emprego. Nas amostras portuguesas, as estruturas fatoriais dos itens e as correlações das escalas vão no mesmo sentido das obtidas no Brasil (Teixeira et. al., 2011).

A estatística inferencial não mostra diferenças significativas entre os resultados médios dos grupos por tipo de escola e sexo. Nesse estudo, os alunos do ensino médio, independentemente de frequentarem ensino regular ou técnico apresentaram crenças equivalentes de autoeficácia em desenvolvimento da carreira. É importante ressaltar, que os alunos de ambas as instituições apresentaram um nível de autoeficácia em desenvolvimento da carreira acima do ponto médio teórico da escala, sugerindo que os alunos estão autoconfiantes face à transição escola-trabalho. $\mathrm{Na}$ pesquisa de Aguilera (2011), as variáveis ligadas à escola (por exemplo, tipo de ensino frequentado), e, muito em particular, a variável que diferenciava tipos de intervenção no apoio aos alunos, mostravam ser altamente discriminativas para o grau de confiança dos alunos em lidar com as tarefas da carreira.

Nesse sentido, apesar da qualidade de ensino e do apoio oferecidos pelas duas instituições não ter sido objeto de análise da presente investigação, considera-se que ela também pode ter contribuído para os resultados, atuando como uma fonte vicária de crenças de autoeficácia. No entanto, para a verificação dessas hipóteses faz-se necessária a realização de outras investigações com vista a confrontar as crenças de autoeficácia com os resultados dos desempenhos, bem como a identificar as fontes das próprias crenças.

Em relação à variável sexo, também não foram encontradas diferenças significativas entre moças e rapazes na percepção da autoeficácia em desenvolvimento de carreira, o que sugere que ambos os grupos apresentam percepções semelhantes quanto às suas capacidades nesse domínio. O estudo de Aguillera (2013) também não apresentou diferenças em função do sexo. Por outro lado, os rapazes revelaram mais confiança em si próprios para lidar com questões relacionadas ao gênero na carreira, no estudo de Lopes (2010).

A análise da autoeficácia em desenvolvimento de carreira em função do nível socioeconômico familiar indica que os alunos de nível socioeconômico familiar mais elevado consideram-se mais capazes de planejar suas carreiras, de se preparar para a busca de emprego e de empreender ações na procura de emprego, do que os alunos de níveis socioeconômicos mais baixos, corroborando os achados de Huang e Hsieh (2011), no contexto chinês, e com adolescentes universitários. A variável nível socioeconômico parece influenciar a percepção da autoeficácia em diferentes domínios do desenvolvimento de carreira. A avaliação da autoeficácia em função do nível socioeconômico familiar tende a evidenciar o efeito da aprendizagem social nas crenças de autoeficácia, e os dados apontam maior autoeficácia em jovens de famílias com mais recursos. A variável nível socioeconômico familiar é complexa. Por um lado, proporciona modelos diferenciados, e, por outro, afeta a percepção das crenças de autoeficácia de carreira em função das condições de acessibilidade às oportunidades educacionais e culturais, às fontes de informações profissionais, entre outras variáveis que podem influenciar os processos de desenvolvimento acadêmico e de carreira. Esses dados confirmam que a familia é um contexto critíco de desenvolvimento, pelas oportunidades de experiências que propicia e pela virtualidade dos modelos que proporciona, duas das fontes de crenças de autoeficácia propostas pela teoria sociocognitiva (Bandura, 1977, Bussey \& Bandura, 1999).

Apesar dos resultados apresentados evidenciarem propriedades psicométricas satisfatórias, considera-se a necessidade de efetuar mais estudos com a utilização do CD-SEI, sobretudo, com a utilização de outros procedimentos de análises de dados e grupos populacionais. Nessa linha de dados, sublinha-se a importância de prosseguir estudos no Brasil e em Portugal para aceder aos indicadores da natureza intercultural do CD-SEI e do próprio construto da autoeficácia aplicada ao domínio da carreira.

Quanto às limitações do estudo, pontuam-se as dificuldades no que se refere ao tamanho da amostra, que não é representativa da população do ensino médio do Brasil, o que limita a generalização dos resultados. Destaca-se, contudo, que os dados deste estudo evidenciam algumas contribuições para a prática, no sentido de as intervenções contemplarem ações para o desenvolvimento das competências da construção da carreira: planejamento, preparação para busca de emprego, procura de emprego e definição de objetivos, sobretudo com os alunos de nível socioeconômico mais baixo.

Os resultados possibilitam também reflexões a respeito das práticas interventivas, evidenciando a necessidade de se trabalhar temas do desenvolvimento da carreira ao longo da vida e estratégias de enfrentamento das adversidades, a fim de desenvolver a confiança pessoal dos alunos, especialmente dos de nível socioeconômico mais baixo. É oportuno que as intervenções de carreira possibilitem o desenvolvimento de competências para reflexão, avaliação e regulação das crenças de autoeficácia de jovens, para que possam lidar de forma mais 
bem sucedida com as tarefas relativas à construção da carreira e da própria vida. As intervenções devem facilitar a aprendizagem dos adolescentes, de forma a poder lidar com as questões que circunstanciam o domínio do desenvolvimento de carreira, fortalecendo suas crenças nesse domínio. As estratégias para essas intervenções podem ser escolhidas como as que se mostram mais eficazes, e que recorrem a procedimentos de modelagem, de experiência direta e de encorajamento. A oferta de educação qualificada, serviços e programas de educação para a carreira dirigidas para todos, com a preocupação de inclusão dos grupos em desvantagem socioeconômica, configuram-se como alternativas viáveis para desenvolver competências dos jovens, tendo em vista o atendimento a demandas por profissionais proativos $\mathrm{e}$ trabalhadores qualificados para atuarem no mundo do trabalho contemporâneo, mas, sobretudo, para auxiliar pessoas em seus projetos de vida.

\section{Referências}

Aguillera, F. (2013). Projeto de vida e preparação para a carreira de jovens aprendizes: Da realidade a intervenção (Tese de doutorado não publicada). Universidade de São Paulo, Ribeirão Preto, SP, Brasil.

Associação Brasileira de Empresas de Pesquisa [ABEP]. (2012). Critério de Classificação Econômica Brasil. São Paulo, SP: ABEP.

Bandura, A. (1977). Self-efficacy: Toward a unifying theory of behavioral change. Psychological Review, 84(2), 191-215. doi.org/10.1037/0033295X.84.2.191

Bandura, A. (1986). Social foundations of thought and action: A social cognitive theory. Englewood Cliffs, NY, Prentice Hall.

Bandura, A. (1993). Perceived self-efficacy in cognitive development and functioning. Educational Psychologist, 28(2), 117-148. doi. org/10.1207/s15326985ep2802_3

Bandura, A. (1997). Self-efficacy: The exercise of control. New York, NY: W. H. Freeman.

Bandura, A. (2001). Social Cognitive Theory: An Agentic Perspective. Annual Reviews Psychologist, 52, 1-26. doi.org/10.1146/annurev.psych.52.1.1

Betz, N. E., \& Hackett, G. (1983). The relationship of mathematics self-efficacy expectations to the selection of science-based college majors. Journal of Vocational Behavior, 23(3), 329-345. DOI: 10.1016/0001-8791(83)90046-5

Betz, N. \& Luzzo, D. (1996). Career assessment and the Career Decision Self-efficacy Scale (CDMSE). Journal of Career Assessment, 4(4), 413-428. doi: 10.1177/106907279600400405

Bussey, K., \& Bandura, A. (1999). Social cognitive theory of gender development and differentiation. Psychological Review, 106(4), 676-713.

Guerreiro-Casanova, D. C., \& Polydoro, S. A. J. (2011). Autoeficácia na formação superior: percepções durante o primeiro ano de graduação. Psicologia: Ciencia e Profissão, 31(1), 50-65.

Huang, J., \& Hsieh, H. (2011). Linking socioeconomic status to social cognitive career theory factors: A partial least squares path modeling analysis. Journal of Career Assessment, 19(4), 452-461. doi.org/ 10.1177/1069072711409723

Leal, M. S. (2013). Autoeficácia percebida em desenbovolviemtno de carreira e interesses profissionais em estudantes do ensino médio regular e técnico. (Dissertação de mestrado não publicada). Universidade de São Paulo, Ribeirão Preto, SP, Brasil.

Lenox, R. A., \& Subich, L. M. (1994). The relationship between self-efficacy beliefs and inventoried vocational interests. The Career Development Quarterly, 42(4), 302-313. doi.org/10.1002/j.2161-0045.1994.tb00514.x

Lent, R. (2005). A social cognitive view of career development and counseling. Em S. D. Brown, \& R. W. Lent (Eds.), Career development and counseling: Putting theory and research to work (pp. 101-127). Hoboken, NJ: Wiley.

Lent, R. W., \& Brown, S. D. (2008). Social cognitive career theory and subjective well-being in the context of work. Journal of Career Assessment, 16(1), 6-21. doi:10.1177/1069072707305769

Lent, R., Brown, S. D., \& Hackett, G. (1994). Toward a unifying social cognitive theory of career and academic interest, choice and performance. Journal of Vocational Behavior, 45(1), 79-122. doi.org/ 10.1006/jvbe.1994.1027

Lent, R., Brown, S. D., \& Hackett, G. (2002). Social cognitive career theory. Em D. Brown \& Associates (Eds.), Career choice and development (4th Ed., pp. 255-311). New York, NY: Wiley.

Lent, R. W., Brown, S. D., \& Larkin, K. C. (1984). Relation of self-efficacy expectations to academic achievement and persistence. Journal of Counseling Psychology, 31(3), 356-362. doi.org/10.1037/0022-0167.31.3.356

Lopes, A. R. (2010). Projectos vocacionais, crenças de auto-eficacia e expectativas parentais em estudantes do $7^{\circ}$ ano de escolaridade em situação de sucesso/ insucesso escolar (Dissertação de mestrado não publicada). Universidade de Lisboa, Lisboa, Portugal.

Lopes, A. R., \& Teixeira, M. O. (2012). Projetos de carreira, autoeficácia e sucesso escolar em ambiente multicultural. Revista Brasileira de Orientação Profissional, 13(1), 7-14.

Metheny, J., \& McWhirter, E. H. (2013). Contributions of social status and family support to college students' career decision self-efficacy and outcome expectations. Journal of Career Assessment, 21(3), 378-394. doi:10.1177/1069072712475164

Noronha, A. P. P., \& Ambiel, R. A. M. (2008). Fontes de eficácia e interesses profissionais: relação entre pais e filhos. Evaluar, 8, 32-45.

Nunes, M. F. O., \& Noronha, A. P. P. (2009). Auto-eficácia para atividades ocupacionais e interesses profissionais em estudantes do ensino médio. Psicologia: Ciência e Profissão, 29(1), 102-115.

Nunes, M. F. O., \& Noronha, A. P. P. (2011). Associações entre auto-eficácia para atividades ocupacionais e interesses em adolescentes. Psicologia: Reflexão e Crítica, 24(1), 1-9. doi.org/10.1590/S0102-79722011000100002

Pajares, F., \& Olaz, F. (2008). Teoria social cognitiva e auto-eficácia: uma visão geral. Em A. Bandura, R. G. Azzi, \& S. Polydoro, Teoria social cognitiva conceitos básicos (pp. 97-114). Porto Alegre, RS: Artmed.

Patel, S. G., Salahuddin, N. M., \& O’Brien, K. M. (2008). Career decision-making self-efficacy of Vietnamese adolescents: The role of acculturation, social support, socioeconomic status, and racism. Journal of Career Development, 34(3), 218-240. doi.org/ $10.1177 / 0894845307308488$ 
Super, D. E. (1990). A life-span, life-space approach to career development. Em D. Brown, L. Brooks \& Associates (Eds.), Career choice and development (2nd Ed., pp. 197-261). San Francisco, CA: Jossey-Bass.

Taylor, K. M., \& Betz, N. E. (1983). Applications of self-efficacy theory to the understanding and treatment of career indecision. Journal of Vocational Behavior, 22(1), 63-81. doi: 10.1016/0001-8791(83)90006-4

Teixeira, M. O. (2007). As crenças de eficácia académica na formação dos interesses e das escolhas vocacionais. Psychologica, 44, 11-23.

Teixeira, M. O. (2008). A escala multidimensional de auto-eficácia percebida: um estudo exploratório numa amostra de estudantes do ensino superior. Revista Iberoamericana de Diagnóstico y Evaluación Psicológica, 25(1), 141-157.

Teixeira, M. O. (2009). Uma medida de auto-eficácia percebida em contextos sociais e académicos. Psychologica, 51, 47-55.

Teixeira, M. O., Barreira, C., Faria, I., Baptista, J., \& Pimentel, A. L. (2011). Inventario de Auto-Eficacia para o Desenvolvimento de Carreira (CD-SEI). Atas do Congresso Iberoamericano de Avaliacao/Evaluacion Psicologica, 8; Conferencia Internacional Avaliação Psicológica: Formas e Contextos, 15. Recuperado de www.researchgate.net/.../00b7d5295ced4ac4df000000.pdf

Yuen, M., Gysbers, N., Chan, R. M. D., Lau, P. S. Y., Leung, T. K. M., Hui, E. K. P., \& Shea, P. M. K. (2005). Developing a career development self-efficacy instrument for Chinese adolescents in Hong Kong. International Journal for Educational and Vocational Guidance, 5(1), 57-73. doi.org/10.1007/s10775-005-2126-3

Yuen, M., Gysbers, N. C., Hui, E. K. P., Leug, T. K. M., Lau, P. S. Y., Chan, R. M. C., \& Ke, S. Y. (2004). Career development self-efficacy inventory: Users' manual. Hong Kong, China: The University of Hong Kong.

Zimmerman, B. J. (2000). Self-efficacy: An essential motive to learn. Contemporary Educational Psychology, 25(1), 82-91. doi:10.1006/ ceps.1999.1016

\section{Sobre as autoras}

Mara de Souza Leal é Psicóloga, Mestre em Psicologia pela Faculdade de Filosofia, Ciências e Letras de Ribeirão Preto.

Lucy Leal Melo-Silva é Psicóloga, Livre Docente do Programa de Pós-Graduação em Psicologia da FFCLRP-USP. Editora Associada da Revista Brasileira de Orientação Profissional e pesquisadora do CNPq.

Maria Odília Teixeira é Psicóloga, Professora Auxiliar da Universidade de Lisboa. É membro do Centro de Investigação em Psicologia da Universidade de Lisboa. 